\title{
OH MASERS IN OUTFLOW REGIONS
}

\author{
M. D. GRAY, D. FIELD AND R. C. DOEL \\ School of Chemistry \\ University of Bristol \\ Cantock's Close \\ Bristol BS8 1TS \\ U.K.
}

ABSTRACT. We combine a sophisticated model of maser propagation with a simple model of an accelerating molecular outflow and show that the observation of different $\mathrm{OH}$ maser frequencies is consistent with emission from different parts of the outflow.

\section{Introduction}

A combination of data on W3(OH) from Gaume \& Mutel (1987) at $1720 \mathrm{MHz}$, Fouquet \& Reid (1982) at 1665 and $1720 \mathrm{MHz}$ and Baudry et. al. (1988) at $4765 \mathrm{Mhz}$ show spatial coincidence of maser spots at these three frequencies. Demagnetized velocities for the strongest emission are: $-42.0 \mathrm{kms}^{-1}(1665 \mathrm{MHz}) ;-43.2 \mathrm{kms}^{-1}(4765 \mathrm{MHz})$ and $-43.6 \mathrm{kms}^{-1}$ $(1720 \mathrm{MHz})$.

We attempt to model these observations as emission from different parts of an accelerating outflow from a young stellar object (YSO) by propagating model masers for short distances under different physical conditions which we calculate to be present along the flow.

\section{The Model}

\subsection{MASER RADIATION TRANSPORT}

Propagation of the partially coherent maser radiation is treated according to the semiclassical theory of Field and Gray (1988). This theory includes the processes of saturation and competitive gain, where different masers compete for the same molecular level populations. 


\subsection{PUMPING}

The masers are assumed to be pumped by a combination of kinetic collisions, background continuum radiation from dust and by FIR line radiation, subject to line overlap, which is treated according to the theory of Doel, Gray and Field (1990). This theory parameterises overlap in terms of a constant velocity gradient and an 'overlap length', essentially a distance over which the velocity gradient is maintained. Transport of the pumping radiation is treated under the Sobolev (LVG) approximation (e.g. Castor 1970).

\subsection{OUTFLOW}

We model the outflow very simply, assuming it to be a plane-parallel flow, with a constant, accelerative velocity gradient in the direction of the flow. Model parameters such as the molecular hydrogen number density and the kinetic temperature are close to consistent with a Joule - Thomson expansion of the gas. The proportion of $\mathrm{OH}$ is assumed constant throughout and equality of the gas and local dust temperatures is maintained.

In a constant velocity gradient model, velocity shift along the outflow is proportional to distance along the outflow; the velocity shift in turn controls the amount of overlap. Increasing distance dilutes the radiation from an external dust component which is associated with the YSO.

\section{Results}

\subsection{CONDITIONS}

Table 1 shows the two sets of model parameters corresponding to positions $\mathrm{A}$ and $\mathrm{B}$ in the flow (see Figure 1) and a third set for a case with no FIR line overlap (see Figure 2).

\begin{tabular}{|l|r|r|r|}
\hline \multicolumn{4}{|c|}{ Table 1. Physical Conditions } \\
\hline Parameter & Region A & Region B & Figure 2 \\
\hline $\mathrm{H}_{2}$ number density & $6.0 \times 10^{6} \mathrm{~cm}^{-3}$ & $3.0 \times 10^{6} \mathrm{~cm}^{-3}$ & $6.0 \times 10^{6} \mathrm{~cm}^{-3}$ \\
External Dust Temperature & $200 \mathrm{~K}$ & $200 \mathrm{~K}$ & $100 \mathrm{~K}$ \\
External Dust Dilution & 0.25 & 0.025 & 0.25 \\
Local Dust Temperature & $150 \mathrm{~K}$ & $75 \mathrm{~K}$ & $50 \mathrm{~K}$ \\
Kinetic Temperature & $150 \mathrm{~K}$ & $75 \mathrm{~K}$ & $50 \mathrm{~K}$ \\
OH Proportion & $10 \mathrm{ppm}$ & $10 \mathrm{ppm}$ & $10 \mathrm{ppm}$ \\
Velocity Shift & $0.8 \mathrm{kms}^{-1}$ & $2.5 \mathrm{kms}^{-1}$ & $0.0 \mathrm{kms}^{-1}$ \\
\hline
\end{tabular}

The conditions A represent an early point in the flow, relatively close to the YSO, while 

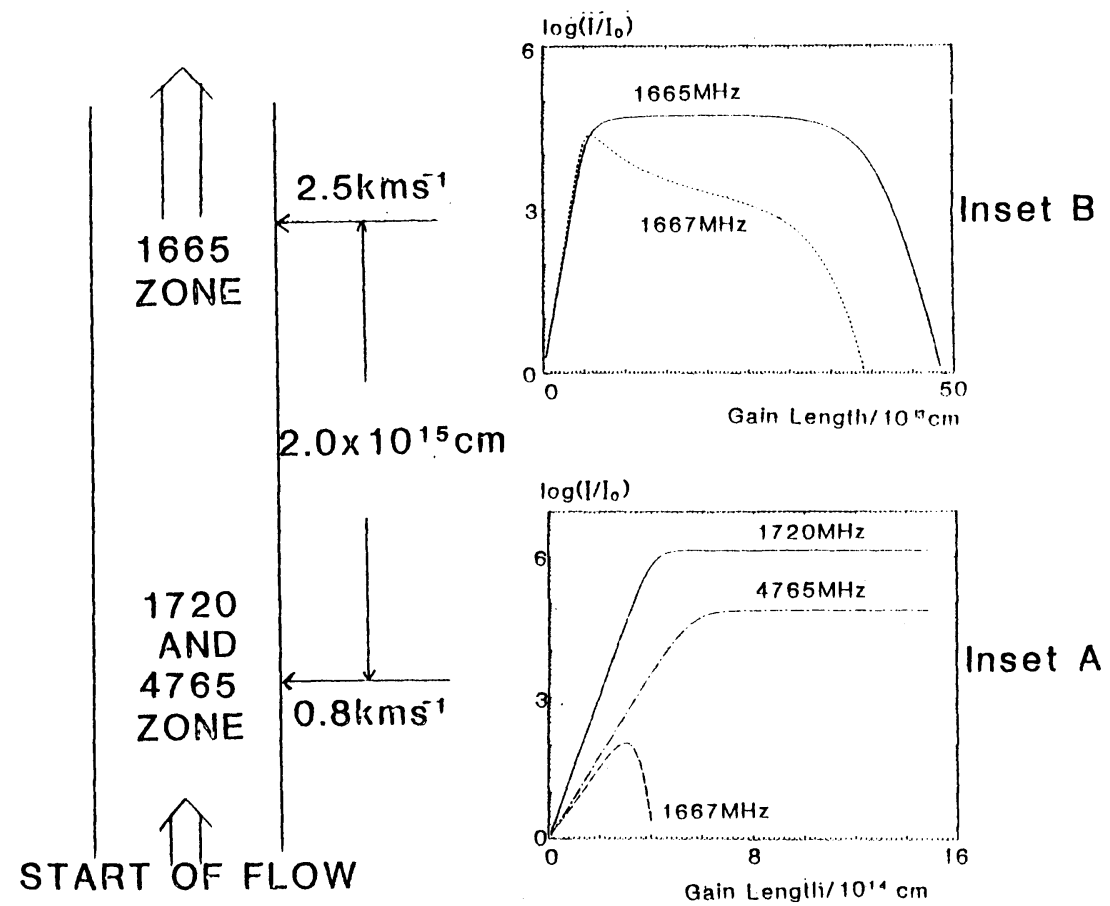

Figure 1: A schematic view of the flow, with maser growth curves appropriate to regions A and $B$ inset

the conditions $\mathrm{B}$ correspond to a more distant region, with considerably more overlap.

\subsection{ACTIVE MASERS}

We see that at point $\mathrm{A}$ we get strong maser emission in the lines $1720 \mathrm{MHz}$ and 4765 $\mathrm{MHz}$ while at point $\mathrm{B}$, we see only $1665 \mathrm{MHz}$ strongly (insets $\mathrm{A}$ and $\mathrm{B}$ in Figure 1). Note that strong $1667 \mathrm{MHz}$ may readily form in the absence of line overlap (Figure 2) but it falls swiftly back into absorption in both sets of conditions $\mathrm{A}$ and $\mathrm{B}$.

\section{Discussion and Conclusion}

The observed spatial correlation in the plane of the sky between masers at $1665 \mathrm{MHz}$, $1720 \mathrm{MHz}$ and $4765 \mathrm{MHz}$ is clearly reproduced by our model. We have also shown that the 


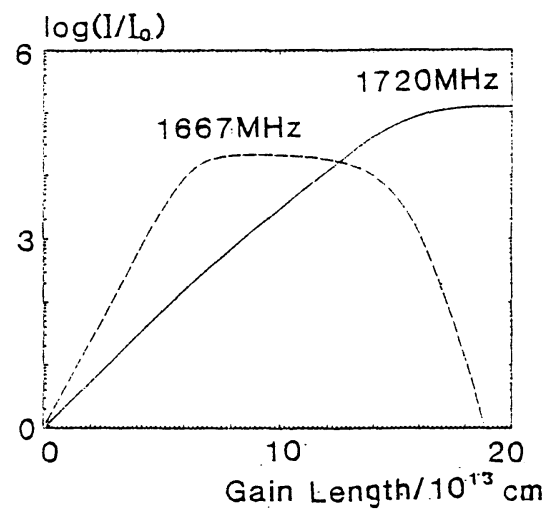

Figure 2: A maser growth curve showing strong emission at $1667 \mathrm{MHz}$. Conditions are given in column 4 of Table 1.

$1665 \mathrm{MHz}$ emission appears from a physically different region at a different velocity from the other two lines again as observed. A general conclusion may be drawn that strong 1665 $\mathrm{MHz}$ spots are indicative of outflows whereas $1667 \mathrm{MHz}$ spots are indicative of regions in which well defined velocity fields are absent.

\section{References}

Baudry, A., Diamond, P., Booth, R., Graham, D. and Walmsley, C. M. 1988. Astron. \& Astrophys., 201, 105.

Castor, J. I.

1970. Mon. Not. Roy. Astron. Soc., 149, 111.

Doel, R. C., Gray M. D. and Field, D.

1990. Mon. Not. Roy. Astron. Soc., 244, 504.

Field, D. and Gray M. D.

1988. Mon. Not. Roy. Astron. Soc., 234, 353.

Fouquet, J. E. and Reid, M. J.

1982. Astronomical Journal, 87, 691.

Gaume, R. A. and Mutel, R. L.

1987. Ap. J. Supp. 65, 193. 